\title{
An investigation of indicators for controlling the quality of a fixture
}

\author{
D. Duret, A. Sergent, and H. Bui-Minh* \\ SYMME Laboratory, University of Savoie, 5 chemin de Bellevue, 74944 Annecy-le-Vieux, France
}

Received: 2 July 2010 / Accepted: 1st September 2010

\begin{abstract}
The quality of fixtures plays an important role in product quality during manufacturing, measuring or assembly. Finding methods for evaluating a fixture's quality, which may depend on workpiece errors, fixture errors, influences of clamping force, friction, etc., is necessary to improve the product quality. This paper proposes the indicators that are used for estimating the quality of a fixture based on workpiece localization repeatability. Here, the workpiece localization will be considered in the following different cases: (1) considering only the influence of different geometric parameters (types) of the fixture, (2) taking into account the clamping force in the different types of the fixture, (3) the influence of friction on the contacts of the workpiece-fixture. A fixture model is presented and analysed with some examples. An experimental fixture and workpiece are then used to analyse and compare with the theoretical results.
\end{abstract}

Keywords: Localization repeatability; clamping force; friction; fixture; indicator

\section{Introduction}

A fixture is a device for positioning and holding a workpiece in the desired location during a machining operation or assembly. A fixturing system usually involves two steps: locating the workpiece in the right position and keeping the workpiece stable during the operations.

A workpiece that is fixed on a fixture must satisfy a unique position, orientation, and static equilibrium. If it deviates from its required location, it is mostly due to the following reasons:

- Deformations of a workpiece, fixture,

- Clamping force,

- Friction between a workpiece and a fixture,

- Cutting force (in a manufacturing operation).

In addition, locator scheme is a vital factor that needs to be considered in fixture layout. In particular, the workpiece should not be hyperstatic.

Many works on fixturing analysis can be found. Wang et al. [1] presented the method used to optimize a locator layout based on the criteria of workpiece repeatability and location accuracy. Clamping optimization is used to minimize the clamping force while satisfying the stability requirement was also described. Thus, the stability requirement is an important condition that needs to be considered for a good fixture. It has been intensively investigated in $[2,3]$. A method that was proposed by

\footnotetext{
* Correspondence:

\{daniel.duret, alain.sergent, hien.bui-minh\}@univ-savoie.fr
}

DeMeter [4] applies restraint analysis to a fixture, which relies on frictionless or frictional surface contact.

This paper presents the method used for estimating the quality of a fixture. It is based on the workpiece localization repeatability on the fixture, which is defined as the deviation of the $n$th workpiece location in comparison to the 1st workpiece location (or the theoretical location which is obtained by a CAD model). It is important to note that only one workpiece is used to control the workpiece repeatability ( $n$ times) on the fixture. Localizationrelated studies, Xiong et al. [5] presented a probing strategy for the measurement a reliable workpiece localization where a reliability-analysis method [6] was used to check the localization accuracy with the proposed measurement points. Li and Melkote [7] improved workpiece location accuracy based on the elastic deformation of surface contacts. Wang [8] used the determinant of a locator information matrix, which characterizes the total variance of workpiece positions and orientation, in order to reduce the workpiece positions errors.

Workpiece localization can be influenced by many sources, which may include workpiece errors, fixture errors, clamping force, friction, or cutting force. In this study, three experimental cases for evaluating a workpiece localization repeatability will be considered: (1) changes in geometric parameters of a fixture are considered, (2) the clamping force is also taken into account with the different geometric parameters of a fixture, (3) friction on the contacts of a workpiece-fixture will be assessed with two assumptions: the surface contacts are perfect or have errors. 
Some studies about the influence of clamping force on workpiece location error are available, such as in Raghu and Melkote [9] where an analytical model is presented to predict workpiece location on the 3-2-1 fixture; Chen and Chen [10] showed the effect of clamping sequences on the stability of fixturing prismatic part and a model that was used for determining clamping force. Schimmels and Peshkin [11] identified the satisfied condition for forceassembly with friction. Most of the above research considers a prismatic part (the 3-2-1 fixture).

The workpiece (tri-axes) used in this research is an element of a honmokinetic joint. The model fixture of this workpiece is based on the SDT concept. Thanks to this model, the indicators are then proposed: the first indicator is defined by the determinant of a torsor, which corresponds to the contact points on the workpiece; the condition number of the Plücker coordinates matrix is calculated for the second indicator that takes the clamping force into account. Last section shows the influence of the friction at the contact points on the workpiece localization.

The ultimate goal of this paper is to propose the necessary indicators for the estimation, and control a workpiece localization repeatability on a fixture. These indicators are useful for choosing a better fixture, i.e. locator position. Furthermore, the methods that are used in the kinematic analysis of a fixture can be integrated into software that can be used for optimizing a fixture in manufacturing, measuring, or assembly.

\section{A geometric model of a fixture}

The geometric model of this fixture is based on the Small Displacement Torsor (SDT) concept $[12,13]$. A SDT is expressed using two vectors: vector $\overrightarrow{\Omega_{S / R}}$ includes three small rotations $(\alpha, \beta, \gamma)$ and vector $\overrightarrow{D_{O}}$ includes three small translations $(u, v, w)$ in a coordinate system $(O x y z)$.

\subsection{Displacement measurement}

A solid, unconstrained in space, has three translations and three rotations. It has six degrees of freedom.

In this research, the displacement of a part on a fixture characterizes the assembly of positions that is near the target position. If displacements are small (compared with its geometric dimension), the geometric transformation that moves from a target position to an actual position (or vice versa) can be modelled by a SDT (1).

$$
\{\mathbf{D}\}=\left\{\overrightarrow{\Omega_{S / R}} \overrightarrow{D_{O}}\right\}_{O}=\left\{\begin{array}{ll}
\alpha & u_{O} \\
\beta & v_{O} \\
\gamma & w_{O}
\end{array}\right\}_{O} .
$$

An overall displacement can be defined by six corresponding scalars. Note that the translational displacement (in $\mathrm{mm}$ ) is modelled by a torque field. The location where it is expressed must be clearly indicated.

$$
\overrightarrow{D_{P_{i}}}=\overrightarrow{D_{O}}+\overrightarrow{\Omega_{S / R}} \wedge \overrightarrow{O P_{i}} .
$$

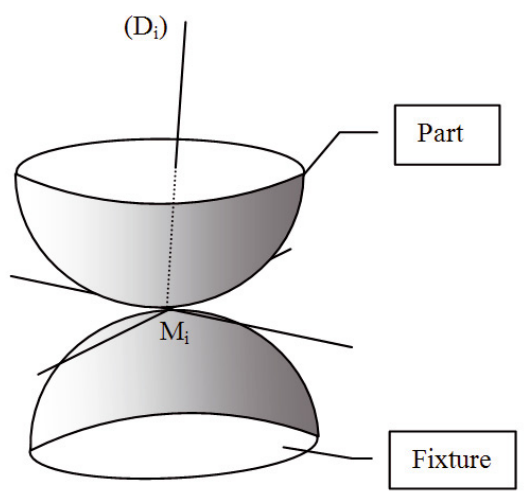

Fig. 1. Normal line $\left(D_{i}\right)$ and contact point $M_{i}$.

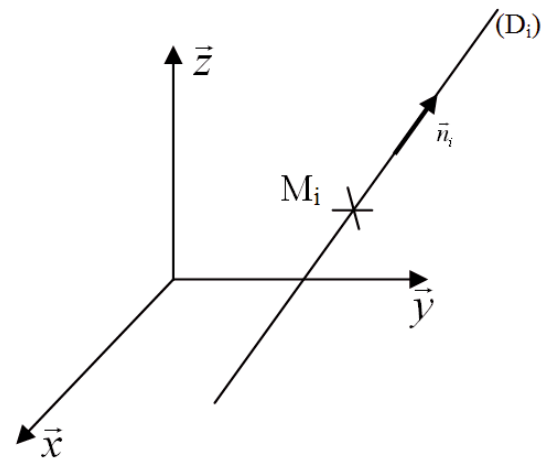

Fig. 2. Line $\left(D_{i}\right)$ in reference $R$.

It can be rewritten in matrix form:

$$
\left[\begin{array}{l}
u_{P_{i}} \\
v_{P_{i}} \\
w_{P_{i}}
\end{array}\right]=\left[\begin{array}{l}
u_{0} \\
v_{0} \\
w_{0}
\end{array}\right]+\left[\begin{array}{lll}
0 & -\gamma & \beta \\
\gamma & 0 & -\alpha \\
-\beta & \alpha & 0
\end{array}\right]\left[\begin{array}{l}
x_{P_{i}} \\
y_{P_{i}} \\
z_{P_{i}}
\end{array}\right]
$$

The measurement of the localization quality depends on these six magnitude scalars. The choice of the contact points of workpiece-fixture pair will strongly influence this measurement.

\subsection{Plücker line coordinate}

From a contact point and a tangent plane, a normal line $\left(D_{i}\right)$ of this plane can be constructed. It passes through the contact point $M_{i}$ (Fig. 1).

Let $R(O, \vec{x}, \vec{y}, \vec{z})$ be a reference of "machine - fixture", the normal line $\left(D_{i}\right)$ can be defined in the Plücker coordinates (Fig. 2).

$\vec{n}_{i}$ is a unit vector of $\left(D_{i}\right)$, so:

$$
\overrightarrow{n_{i}}=\left(n_{x i}, n_{y i}, n_{z i}\right) \text { and } \overrightarrow{O M_{i}}=\left(x_{i}, y_{i}, z_{i}\right) .
$$

The vector product can be calculated:

$$
\overrightarrow{g_{O}}=\overrightarrow{O M_{i}} \wedge \overrightarrow{n_{i}}
$$



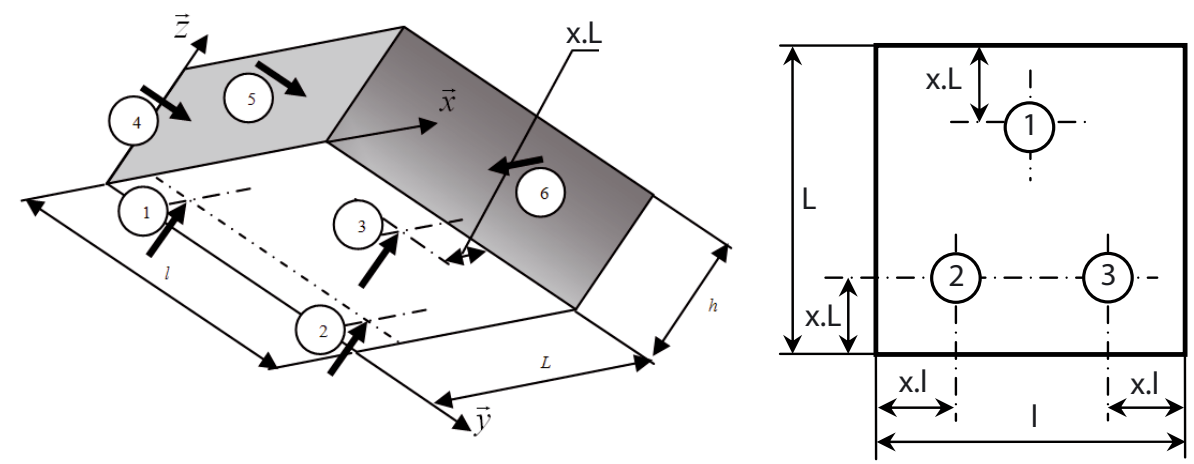

Fig. 3. Influence of the coefficient $x$ on the position.

The Plücker coordinates are defined by six scalars as follows:

$$
\left\{\mathbf{P}_{i}\right\}=\left\{\overrightarrow{n_{i}} \overrightarrow{O M_{i}} \wedge \vec{n}_{i}\right\}_{O}=\left\{\begin{array}{ll}
n_{x i} & y_{i} n_{z i}-z_{i} n_{y i} \\
n_{y i} & z_{i} n_{x i}-x_{i} n_{z i} \\
n_{z i} & x_{i} n_{y i}-y_{i} n_{x i}
\end{array}\right\}_{O} .
$$

These six components are dependent, and have the following relationship:

$$
\left\{\begin{array}{l}
n_{x i}^{2}+n_{y i}^{2}+n_{z i}^{2}=1 \\
\vec{n}_{i} \bullet \vec{g}_{0}=0
\end{array} .\right.
$$

So,

$$
\begin{aligned}
n_{x i}\left(y_{i} n_{z i}-z_{i} n_{y i}\right)+n_{y i} & \left(z_{i} n_{x i}-x_{i} n_{z i}\right) \\
& +n_{z i}\left(x_{i} n_{y i}-y_{i} n_{x i}\right)=0 .
\end{aligned}
$$

Note: point $M_{i}$ may be taken anywhere on the normal line $\left(D_{i}\right)$.

\subsection{Rank of a line system}

The six normal lines $\left(D_{1}\right)$ to $\left(D_{6}\right)$ set up a line system. The order of the largest nonzero determinants, which can be extracted the following matrix (9) from the Plücker line coordinates, belongs to the line system. It is named rank of a line system, notation $r$.

$$
r=\left|\begin{array}{llllll}
n_{x 1} & n_{x 2} & n_{x 3} & n_{x 4} & n_{x 5} & n_{x 6} \\
n_{y 1} & n_{y 2} & n_{y 3} & n_{y 4} & n_{y 5} & n_{y 6} \\
n_{z 1} & n_{z 2} & n_{z 3} & n_{z 4} & n_{z 5} & n_{z 6} \\
y_{1} n_{z 1}-z_{1} n_{y 1} & g_{O, x 2} & g_{O, x 3} & g_{O, x 4} & g_{O, x 5} & y_{6} n_{z 6}-z_{6} n_{y 6} \\
z_{1} n_{x 1}-x_{1} n_{z 1} & g_{O, y 2} & g_{O, y 3} & g_{O, y 4} & g_{O, y 5} & z_{6} n_{x 6}-x_{6} n_{z 6} \\
x_{1} n_{y 1}-y_{1} n_{x 1} & g_{O, z 2} & g_{O, z 3} & g_{O, z 4} & g_{O, z 5} & x_{6} n_{y 6}-y_{6} n_{x 6}
\end{array}\right|
$$

Note: the maximum rank of a line system is 6 .

\subsection{Hunt's theorem}

Theorem. Let $r$ be the rank of the line system $\{\mathrm{S}\}$ (normal lines at contacts), the remaining degrees of freedom between two solids are defined using the following equation:

$$
d=6-r
$$

Application. In this research, a workpiece in a fixture is immobilized. Thus, the rank that needs to be obtained is $r=6$.

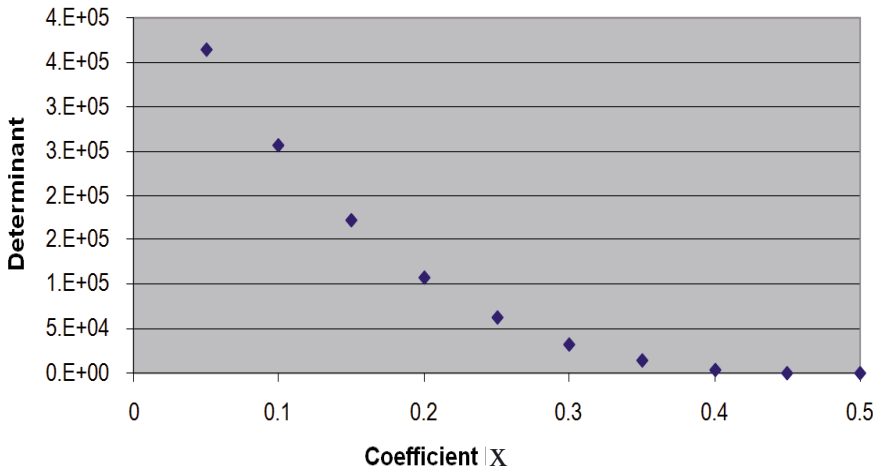

Fig. 4. The determinant is considered as the quality indicator of the fixture.

\section{First indicator proposed}

Thanks to the matrix of the Plücker line coordinates, the measurement of the fixture quality will be performed based on a value of an associated determinant.

\subsection{Example 1: Kelvin's fixture}

The basic fixture (plan-line-point) will be used to consider the influence of the coefficient $x$ (Fig. 3 ) on a determinant of the constructed matrix in the Plücker coordinates.

The lateral locating points are halfway up to the workpiece. Let $L=100 \mathrm{~mm}, l=50 \mathrm{~mm}, h=20 \mathrm{~mm}$ be the dimensions of the workpiece, Figure 4 shows the relation of coefficient $x$ and the determinants which are obtained by matrices of 6 locating points as in equation (9).

If coefficient $x$ equals 0.5 , there are only 3 effective supports. It becomes a spherical joint (3 degrees of freedom).

This technique allows us to compare different solutions, which depend on a nearby space.

\subsection{Example 2: Boys' fixture}

The following fixture, namely Boys' fixture (Fig. 5), will be analysed to evaluate the influence of geometric parameters on its quality. 


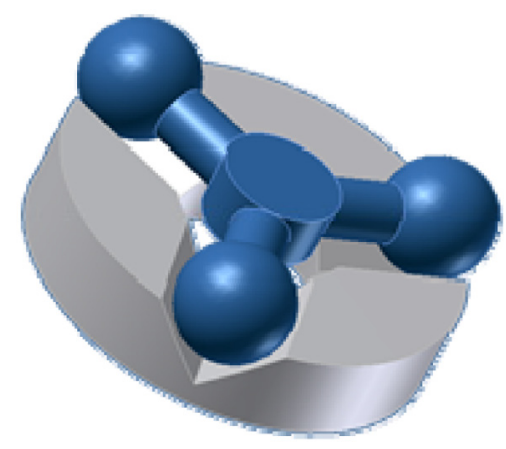

Fig. 5. Boys' fixture.

There are two geometric parameters of this fixture: angle of $\mathrm{V}$-Blocks and radius of contact points, which is calculated from the centre to a contact point, notation $V$ and $R$.

Let $V=90^{\circ}$ and $R=50 \mathrm{~mm}$ be geometric parameters of a fixture, six components of the Plücker coordinates can be obtained as in Table 1.

Figure 6 shows determinants, which are obtained by matrices of 6 locating point as in equation (9), of different configurations of fixtures.

Note: similarly, the calculation of another indicator is also possible to define the measurement, which uses the norm of the matrix of the Plücker coordinates [14], to control the quality of the contacts. For example the pseudo-Euclidean norm can be used to estimate this other indicator.

$$
\|\|_{1} \leqslant\|\|_{E} \leqslant\|\|_{2}
$$

with

$$
\|P\|_{E}=\sqrt{\sum_{i} \sum_{j} p_{i j}^{2}} .
$$

The elements of this matrix are not homogeneous, but the square norms of the normal lines are always equal to 1 .

As we have seen in the two above examples, values of determinants are high when distances of locating points are large. In other words, the higher the value of a determinant, the better the quality of a fixture. This proposition is used to confirm the following experimental application.

\section{Validation by an experimental approach}

For each new location of the workpiece (chronological order), the coordinates of 6 points $\left(M_{1}\right.$ to $\left.M_{6}\right)$ (Fig. 7) on the workpiece will be measured by the Coordinate Measuring Machine $(\mathrm{CMM})$.

\subsection{Determination of positioning deviations}

A CAD model is created to initialize the measurement points $M_{i}$ on the workpiece. The first location of the workpiece on the fixture (or the theoretical location that is obtained by a CAD model) will be used as a reference (0), it corresponds to:

$$
\overrightarrow{O M_{i 0}}=\left(x_{i 0}, y_{i 0}, z_{i 0}\right) .
$$

A new workpiece location, which is defined after each disassembly and reassembly of the workpiece on the fixture, is measured using the same program (six measurement points $M_{1}$ to $M_{6}$ ). The $k$ th measurement of the workpiece location is shown as follows:

$$
\overrightarrow{O M_{i k}}=\left(x_{i k}, y_{i k}, z_{i k}\right)
$$

The $k$ th workpiece location compares with reference 0 as follows:

$$
\overrightarrow{D_{M i 0 k}}=\overrightarrow{M_{i 0} M_{i k}}=\left(x_{i k}-x_{i 0}, y_{i k}-y_{i 0}, z_{i k}-z_{i 0}\right)
$$

let

$$
\delta_{i k}=\overrightarrow{n_{i}} \bullet \overrightarrow{D_{M i 0 k}}
$$

$$
\delta_{i k}=n_{i x}\left(x_{i k}-x_{i 0}\right)+n_{i y}\left(y_{i k}-y_{i 0}\right)+n_{i z}\left(z_{i k}-z_{i 0}\right)
$$

with

$$
\begin{gathered}
\overrightarrow{D_{O k}}=\overrightarrow{D_{M i 0 k}}+\overrightarrow{O M_{i 0}} \wedge \overrightarrow{\Omega_{k}} \\
\overrightarrow{n_{i}} \bullet \overrightarrow{D_{O k}}=\overrightarrow{n_{i}} \bullet \overrightarrow{D_{M i 0 k}}+\overrightarrow{n_{i}} \bullet \overrightarrow{O M_{i 0}} \wedge \overrightarrow{\Omega_{k}} \\
\overrightarrow{n_{i}} \bullet \overrightarrow{D_{O k}}-\left(\overrightarrow{n_{i}}, \overrightarrow{O M_{i 0}}, \overrightarrow{\Omega_{k}}\right)=\delta_{i k} \\
\left\{\mathbf{D}_{\mathbf{k}}\right\}=\left\{\overrightarrow{\Omega_{k, S / R}} \overrightarrow{D_{O k}}\right\}_{O}=\left\{\begin{array}{ll}
\alpha_{k} & u_{O k} \\
\beta_{k} & v_{O k} \\
\gamma_{k} & w_{O k}
\end{array}\right\}_{O}
\end{gathered} .
$$

It is solved using a system of 6 equations ( $i=1$ to 6 ):

$$
\left[\begin{array}{lll}
n_{i x} & n_{i y} & n_{i z}
\end{array}\right]\left[\begin{array}{c}
u_{O k} \\
v_{O k} \\
w_{O k}
\end{array}\right]-\left|\begin{array}{lll}
n_{i x} & x_{i 0} & \alpha_{k} \\
n_{i y} & y_{i 0} & \beta_{k} \\
n_{i z} & z_{i 0} & \gamma_{k}
\end{array}\right|=\delta_{i k}
$$

where $u_{O k}, v_{O k}, w_{O k}, \alpha_{k}, \beta_{k}, \gamma_{k}$ are unknown.

So,

$n_{i x} u_{0 k}+n_{i y} v_{0 k}+n_{i z} w_{O k}+g_{O, i x} \alpha_{k}+g_{O, i y} \beta_{k}+g_{O, i z} \gamma_{k}=\delta_{i k}$

The left side of this equation (23) $\left\{\mathbf{P}_{i}\right\}\{\mathbf{D}\}$ is a product of a SDT and a Plücker coordinates torsor of the normal line at a point $i$.

From the matrix form, the following equations are obtained:

$$
\left[\begin{array}{llllll}
g_{O, x 1} & g_{O, y 1} & g_{O, z 1} & n_{x 1} & n_{y 1} & n_{z 1} \\
g_{O, x 2} & g_{O, y 2} & g_{O, z 2} & n_{x 2} & n_{y 2} & n_{z 2} \\
g_{O, x 3} & g_{O, y 3} & g_{O, z 3} & n_{x 3} & n_{y 3} & n_{z 3} \\
g_{O, x 4} & g_{O, y 4} & g_{O, z 4} & n_{x 4} & n_{y 4} & n_{z 4} \\
g_{O, x 5} & g_{O, y 5} & g_{O, z 5} & n_{x 5} & n_{y 5} & n_{z 5} \\
g_{O, x 6} & g_{O, y 6} & g_{O, z 6} & n_{x 6} & n_{y 6} & n_{z 6}
\end{array}\right]\left[\begin{array}{l}
\alpha \\
\beta \\
\gamma \\
u \\
v \\
w
\end{array}\right]=\left[\begin{array}{l}
\delta_{1} \\
\delta_{2} \\
\delta_{3} \\
\delta_{4} \\
\delta_{5} \\
\delta_{6}
\end{array}\right]
$$

It can be rewritten in the simple form:

$$
[A][\alpha]=[\delta]
$$

or,

$$
[\alpha]=[A]^{-1}[\delta]
$$


Table 1. Six components of the Plücker coordinates.

\begin{tabular}{|c|c|c|c|c|c|c|c|c|c|}
\hline & $\overline{P l}$ & 11 & Pt 2 & Pt 3 & Pt 4 & Pt 5 & Pt 6 & & \\
\hline$x$ & & 50.000 & 50.000 & -25.000 & -25.000 & -25.000 & -25.000 & Radius $R$ : & 50 \\
\hline$Y$ & & 0.000 & 0.000 & 43.301 & 43.301 & -43.301 & -43.301 & $1 / 2$ anole of $V$. & 45 \\
\hline$Z$ & & 0.000 & 0.000 & 0.000 & 0.000 & 0.000 & 0.000 & & \\
\hline$n_{x}$ & & 0.000 & 0.000 & -0.612 & 0.612 & 0.612 & -0.612 & & \\
\hline$n_{y}$ & & 0.707 & -0.707 & -0.354 & 0.354 & -0.354 & 0.354 & & \\
\hline$n_{z}$ & & 0.707 & 0.707 & 0.707 & 0.707 & 0.707 & 0.707 & & \\
\hline & & & & Pt 1 & Pt 2 & Pt3 & Pt 4 & Pt 5 & Pt 6 \\
\hline & & & $n_{i}$ & 0.000 & 0.000 & -0.612 & 0.612 & 0.612 & -0.612 \\
\hline & $\bar{n}_{i}$ & & $n_{y i}$ & 0.707 & -0.707 & -0.354 & 0.354 & -0.354 & 0.354 \\
\hline$\left\{\mathcal{P}_{i}\right\}=$ & & $=$ & $n_{i}$ & 0.707 & 0.707 & 0.707 & 0.707 & 0.707 & 0.707 \\
\hline & & & $-z_{i}, n_{p}$ & 0.000 & 0.000 & 30.619 & 30.619 & -30.619 & -30.619 \\
\hline & $O \bar{M}_{i} \wedge \vec{n}_{i}$ & & $-x_{i}, n_{i}$ & -35.355 & -35.355 & 17.678 & 17.678 & 17.678 & 17.678 \\
\hline & & & $-y_{i} \cdot n_{n}$ & 35.355 & -35.355 & 35.355 & -35.355 & 35.355 & -35.355 \\
\hline
\end{tabular}

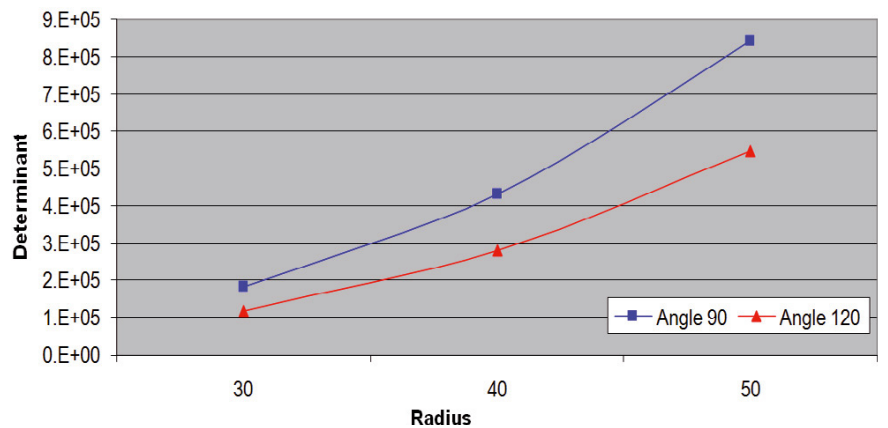

Fig. 6. Theoretical influences of the geometric parameters of the fixture on the determinant.

\subsection{Data treatment}

If $k$ varies from 1 to $n$, the 6 series of $n$ data will be obtained. It may include noises, which is due to many sources (operator, material, method, machine and environment...) in the result obtained.

Firstly, the noise of measurements that is verified is negligible using the 100 measurements of the workpiece on the fixtures without disassembly.

As previously mentioned, the six components $\left(u_{O k}, v_{O k}\right.$, $\left.w_{O k}, \alpha_{k}, \beta_{k}, \gamma\right)$ of the SDT are obtained. The quality of a fixture will be certified by the variability of the unknowns. The variability of each component is estimated using an experimental standard deviation $(s)$ and a confidence interval. The computation of the confidence interval for the standard deviation is given as [15]:

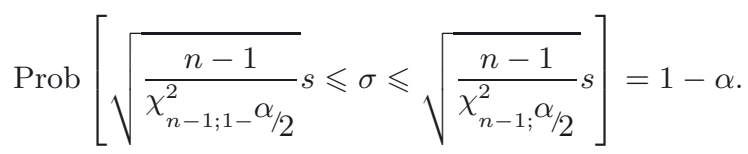

For example, $95 \%$ confidence (or the probability is 0.95 ) for a sample of 100 values gives:

$$
\operatorname{Prob}\left[\sqrt{\frac{99}{\chi_{99 ; 0,975}^{2}}} s \leqslant \sigma \leqslant \sqrt{\frac{99}{\chi_{99 ; 0,025}^{2}}} s\right]=95 \%
$$

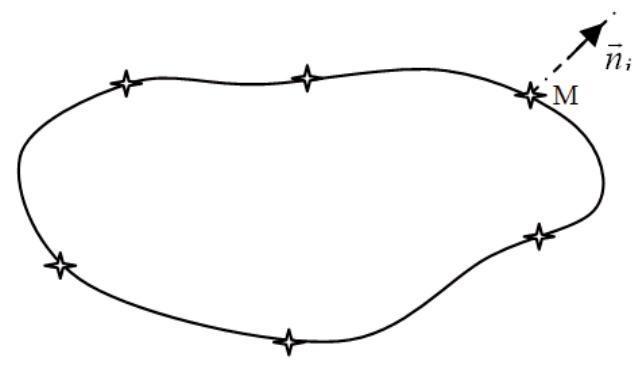

Fig. 7. Choice of 6 measurement points $M_{i}$.

$$
\operatorname{Prob}[0.88 s \leqslant \sigma \leqslant 1.16 s]=95 \% \text {. }
$$

\subsection{Experimental results}

\subsubsection{Experimental fixture}

An experimental fixture (Fig. 8) used to locate and hold the tri-axes workpiece will be measured and analysed to compare with the theoretical results.

The workpiece is fixed on three short V-Blocks in which its angle and its position on the base of the fixture can be changed. The geometric parameters of the fixture and its notations are shown in Table 2.

\subsubsection{Noise of measurement}

A measurement for each fixture configuration was carried out one hundred times on the two CMMs to estimate the noises of measurements. The technical data of the CMMs were used as follows:

- MarVision MS222

The technical data of the probe is used to measure as follows:

- Manufacturer: Renishaw 


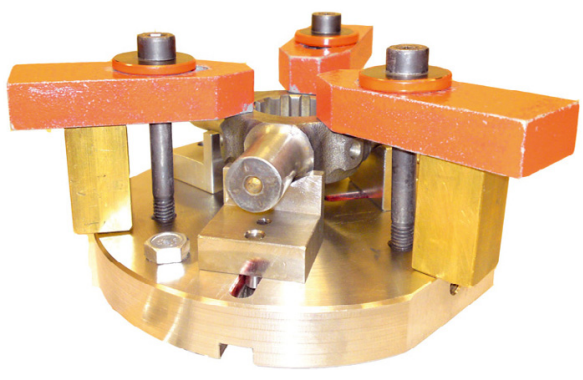

Fig. 8. Experimental fixture.

Table 2. Geometric parameters of the fixture and its notations.

\begin{tabular}{lcc}
\hline & \multicolumn{2}{c}{ Diameter } \\
\cline { 2 - 3 } Angle of V-Block & $67(\mathrm{~mm})$ & $110(\mathrm{~mm})$ \\
\hline $90^{\circ}$ & $\mathrm{V}^{\circ} \mathrm{d}_{\min }$ & $\mathrm{V} 90 \mathrm{D}_{\max }$ \\
$120^{\circ}$ & ${\mathrm{V} 120 \mathrm{~d}_{\min }}$ & $\mathrm{V} 120 \mathrm{D}_{\max }$ \\
\hline
\end{tabular}

- Model: TP200

- Technology: Touch probe

- Precision of probe:

- Unidirectional repeatability $(2 s \mu \mathrm{m}): 0.50 \mu \mathrm{m}$

- $X Y Z$ (3D) form measurement deviation:

- Sip Orion $\pm 1.40 \mu \mathrm{m}$

- Resolution: $0.1 \mu \mathrm{m}$

- Precision: $0.8 \mu \mathrm{m}+L \div 800$.

The results obtained from these measurements show that the noises of the measurements are small. Therefore, it is neglected in the following calculations.

\subsubsection{Analysis of the results obtained}

The variability of the components $\left(u_{O k}, v_{O k}, w_{O k}, \alpha_{k}, \beta_{k}\right.$, $\gamma_{k}$ ) is given by an experimental standard deviation and the associated confidence intervals.

Figures 9 and 10 show the standard deviations of translations and rotations of the different configurations of the fixture. They are used as the indicators to evaluate the quality of the workpiece localizations on the different fixtures.

The results of the theoretical indicator (determinant) in Figure 6 show that:

- the larger the diameter, the higher the determinant,

- the greater the angle, the higher the determinant,

Let us now compare the experimental results and the theoretical indicator (determinant) in Figure 6. It shows that:

- It is right for the fixtures with the V120 parameters, but it is only right for the components $w$ and $\gamma$ of the fixtures with the V90 parameters.

- However, the variability of the experimental fixture with parameter V120 is smaller than the theoretical results.

- For the influences of the different angles of V-Blocks on the quality of the fixture, there is a contradiction between the theoretical and experimental results.
These conclusions can be summed up in the following tables (Tabs. 3 and 4).

\section{Second indicator proposed}

In the above proposition, the quality of the fixture was considered based on the geometric parameters. The different types (different geometric parameters) of the fixture strongly influence on normal forces at contacts between workpiece and fixture. The contacts create local deformation which is nonrepoducible (friction, crushing, micro-adhesion...). The influence of the clamping force will now be taken into account in the indicator that is proposed in this section.

\subsection{Influence of the clamping force}

If the workpiece is in static equilibrium, the actions of the fixture on the workpiece are $\sum \vec{F}_{i}$ and a torsor of external force that is modelled by the clamping force $\vec{P}$ (Fig. 11).

The resultant on the workpiece can be shown as follows:

$$
\begin{gathered}
\sum_{i} F_{i} \cdot \overrightarrow{n_{i}}=-\vec{P} \\
\sum_{i} \overrightarrow{O M_{i}} \wedge F_{i} \cdot \overrightarrow{n_{i}}=-\overrightarrow{O M_{P}} \wedge \vec{P} .
\end{gathered}
$$

It can be rewritten in the following equations:

$$
\text { [Coord_Plück] }\left[\begin{array}{c}
F_{1} \\
F_{2} \\
F_{3} \\
F_{4} \\
F_{5} \\
F_{6}
\end{array}\right]=\left[\begin{array}{l}
-P_{x} \\
-P_{y} \\
-P_{z} \\
-y_{P} P_{z}+z_{P} P \\
-z_{P} P_{x}+x_{P} P \\
-x_{P} P_{y}+y_{P} P
\end{array}\right] .
$$

So,

$$
\left[\begin{array}{l}
F_{1} \\
F_{2} \\
F_{3} \\
F_{4} \\
F_{5} \\
F_{6}
\end{array}\right]=[\text { Coord_Plück }]^{-1}\left[\begin{array}{l}
-P_{x} \\
-P_{y} \\
-P_{z} \\
-y_{P} P_{z}+z_{P} P \\
-z_{P} P_{x}+x_{P} P \\
-x_{P} P_{y}+y_{P} P
\end{array}\right]
$$

In the calculation of $F_{i}$, the angle of V-Block is an important factor (point effect) while there are no effects of the increase of the diameter on the fixture quality. It is possible that the increase of the resultant force and the small defects of local geometry generate non-homogeneous deformations $\left(F_{i}=f\left(\delta_{i}\right)\right)$.

The variability of the calculation of contact forces closely relates to the condition number of a transformation matrix.

The pseudo-condition $K$ (Euclidean) of the matrix "Coord_Plück" can be calculated to be used as a new indicator for the quality of the fixture:

$$
K=\|[\text { Coord_Plück }]\left\|_{E}\right\|[\text { Coord_Plück }]^{-1} \|_{E}
$$

with

$$
\|[\text { Coord_Plück }] \|_{E}=\sqrt{\sum_{i} \sum_{j} C p_{i j}^{2}}
$$




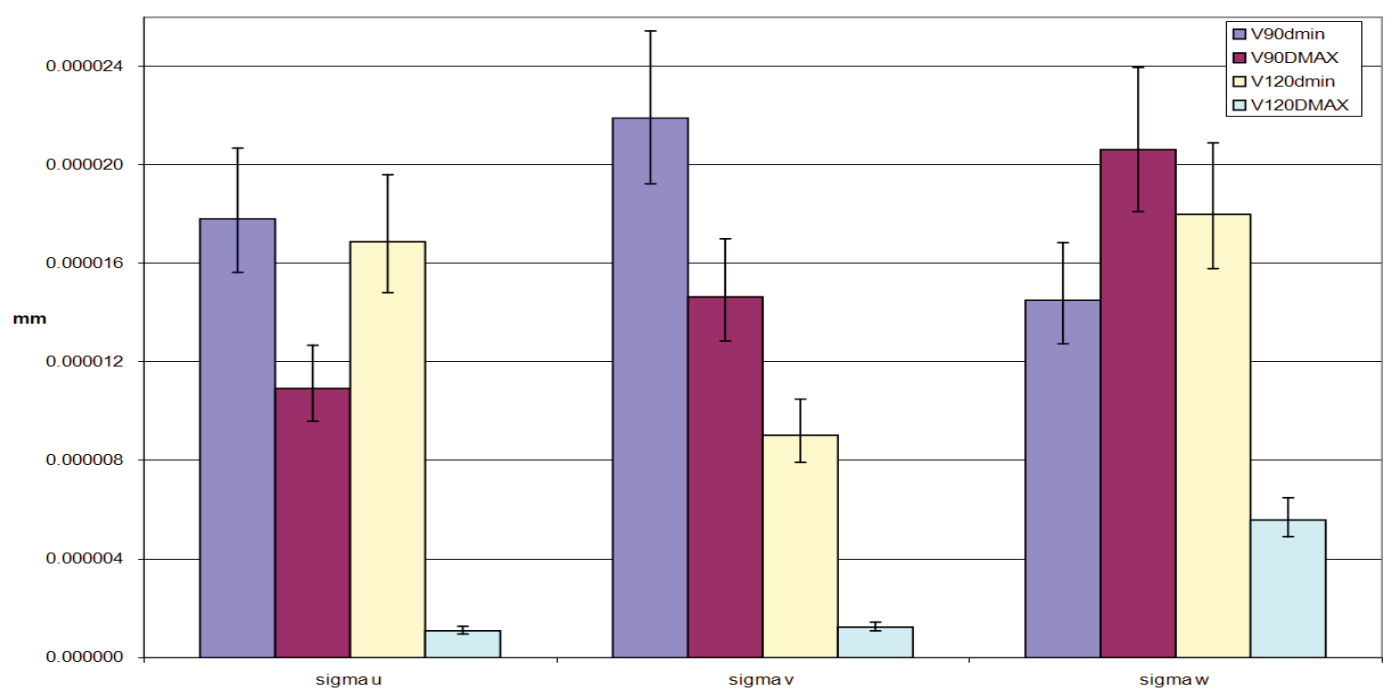

Fig. 9. Indicators of the variability for translations.

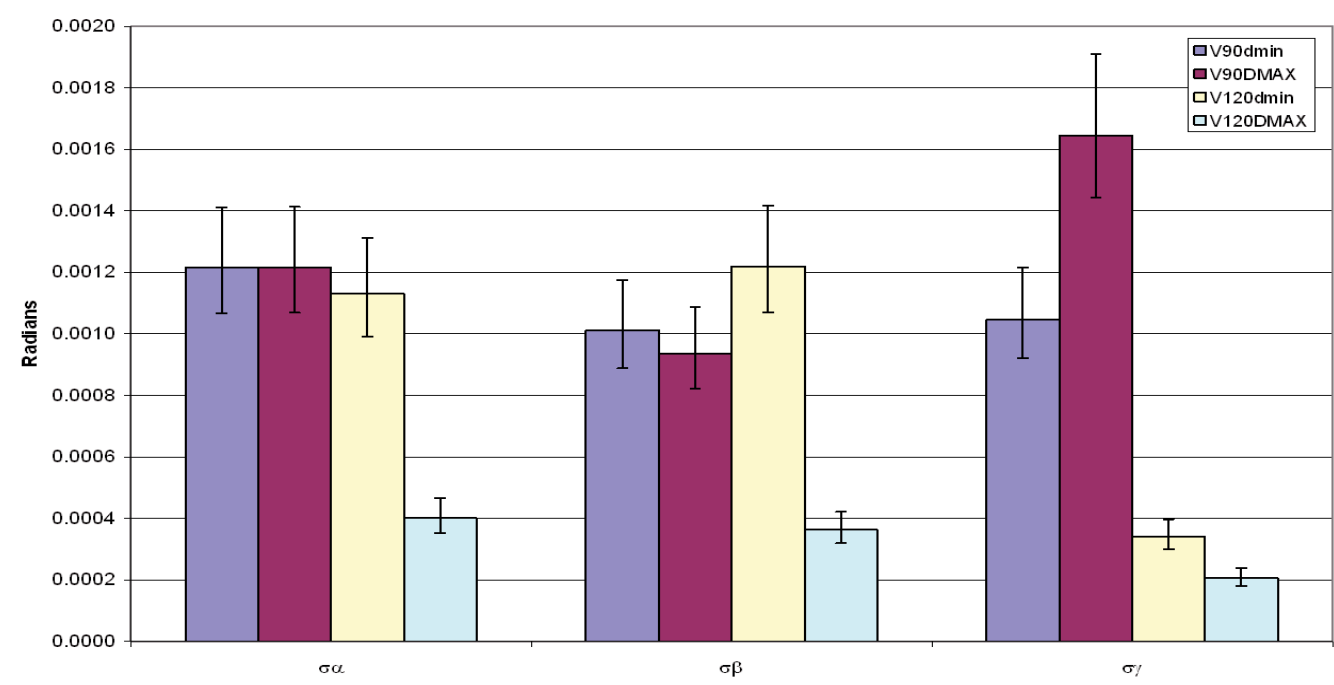

Fig. 10. Indicators of the variability for rotations.

Table 3. Experimental results.

\begin{tabular}{lccc}
\hline \multicolumn{4}{c}{ The first proposed indicator (indeterminant) } \\
\hline Statement & Components & V120 & V90 \\
\hline \multirow{4}{*}{ The larger the diameter, } & $v$ & $\checkmark$ & $\mathbf{x}$ \\
the higher the determinant & $w$ & $\checkmark$ & $\mathbf{x}$ \\
& $\alpha$ & $\checkmark$ & $\mathbf{x}$ \\
& $\beta$ & $\checkmark$ & $\mathbf{x}$ \\
& $\gamma$ & $\checkmark$ & $\checkmark$ \\
\hline
\end{tabular}

$\checkmark$ is suitable for the statement; $\boldsymbol{x}$ is not suitable for the statement.

where $C p_{i j}$ is a element of the Coor_Plück matrix.

Each fixture has a different value of $K$. These calculated values are shown in Figure 12.

The results in Figure 12 show that:
Table 4. Influence of the angle parameter.

\begin{tabular}{lcc}
\hline \multirow{2}{*}{ Statement } & \multicolumn{2}{c}{ Type of fixture } \\
\cline { 2 - 3 } & $\begin{array}{c}\text { Theoretical } \\
\text { fixture }\end{array}$ & $\begin{array}{c}\text { Experimental } \\
\text { fixture }\end{array}$ \\
\hline $\begin{array}{l}\text { The greater the angle, } \\
\text { the higher the determinant }\end{array}$ & $\checkmark$ & $\mathbf{x}$ \\
\hline
\end{tabular}

- the larger the diameter, the higher the coefficient $K$,

- the greater the angle, the higher the coefficient $K$.

\subsection{Analysis of results}

This indicator is mostly consistent with the experimental results. It shows that the $\mathrm{V} 120 \mathrm{D}_{\max }$ fixture, whose parameters are: angle of V-Block at $120^{\circ}$ and the largest diameter, has the 


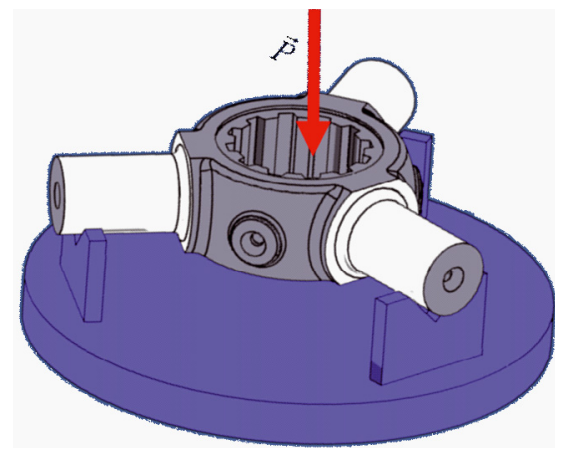

Fig. 11. Clamping force.

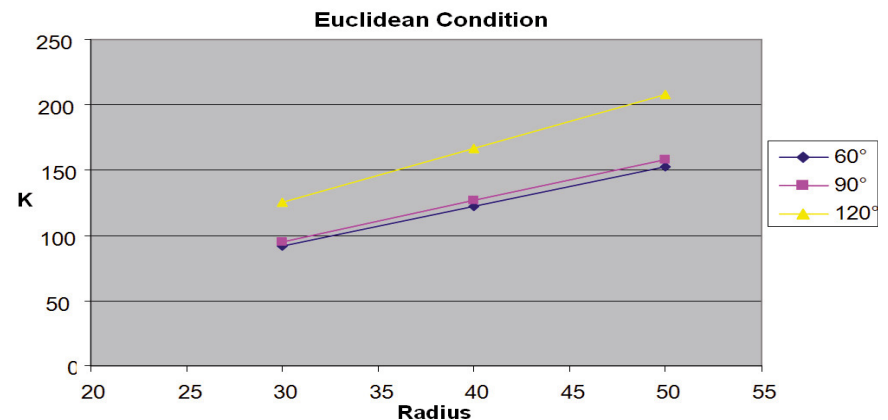

Fig. 12. The Euclidean condition.

smallest variability. Only the components $\gamma$ and $w$ of the V90 contradict the experimental results (Tab. 5).

\section{Taking into account of friction}

The two above indicators were used to quantify the quality of the Boys' fixture. However, these indicators could not completely estimate this experimental fixture. The frictions at the contacts of the workpiece and the fixture can influence the quality of workpiece localization. "Friction is the predominant mechanism for workpiece holding in most fixturing application" [16]. Therefore, the influence of friction will be taken into account for evaluating the quality of the fixture in this section.

We will consider:

- the slipping force that makes the workpiece slipping on the surfaces of V-Block,

- whether the friction force is influenced by geometry on contact points.

\subsection{Modelling}

Each trunnion of the workpiece has 2 contact points $\left(c_{i}\right)$ with the $\mathrm{V}$-Block, and $\overrightarrow{n_{i}}$ is a normal of contact point $i(i \in(1,6))$, so:

- The gravity of the workpiece

$$
\vec{G}=m \cdot \vec{g} .
$$

Table 5. Summary of the conclusions.

\begin{tabular}{lccc}
\hline \multicolumn{4}{c}{ The second proposed indicator $($ coefficient $K$ ) } \\
\hline Statement & Components & V120 & V90 \\
\hline The larger the diameter, & $u$ & $\checkmark$ & $\checkmark$ \\
the higher the coefficient $K$ & $v$ & $\checkmark$ & $\checkmark$ \\
and & $w$ & $\checkmark$ & $\mathbf{x}$ \\
The greater the angle, & $\alpha$ & $\checkmark$ & $\checkmark$ \\
the higher the coefficient $K$ & $\beta$ & $\checkmark$ & $\checkmark$ \\
& $\gamma$ & $\checkmark$ & $\mathbf{x}$
\end{tabular}

$\checkmark$ is suitable for the statement; $\mathbf{x}$ is not suitable for the statement.

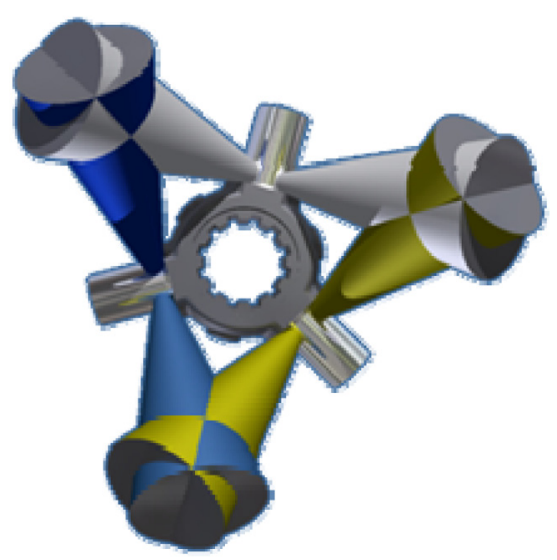

Fig. 13. Friction cones.

- The resultant force of disassembly

$$
\overrightarrow{F_{D}}=\lambda \cdot \vec{z} \text {. }
$$

- The displacement vector $\overrightarrow{\delta_{i}}$.

- The reaction force of the workpiece on the fixture $\left(\vec{F}_{i}\right)$ :

- along the normal $\overrightarrow{n_{i}}$ if the friction coefficient $\tan (\varphi)=0$ $\left(\overrightarrow{F_{D}}=0\right)$

$\circ$ inside the friction cone if the friction coefficient $\tan (\varphi) \neq 0\left(\overrightarrow{F_{D}} \neq \overrightarrow{0}\right)$

where $\tan (\varphi)$ is a friction coefficient.

- An angle of the V-Block: V.

Figure 13 shows the friction cone at the contact points on the workpiece.

There are two situations for the workpiece on the fixture: contact and non-contact. It is shown in Figure 14.

\subsection{Methods}

\subsubsection{First assumption}

Here, the contacts between the workpiece and the fixture are considered perfect (Fig. 14). It means that there is no interpenetration between the workpiece and the fixture, and the surface defects are neglected. Hence, there is no interlock between 

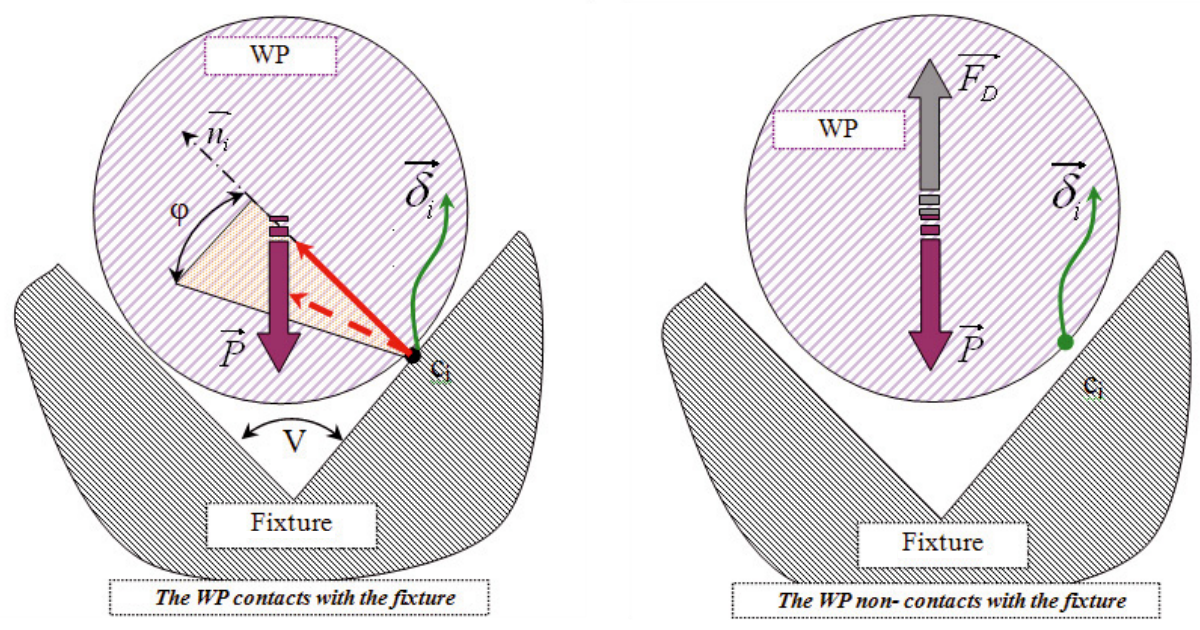

Fig. 14. Two situations of the workpiece on the fixture.

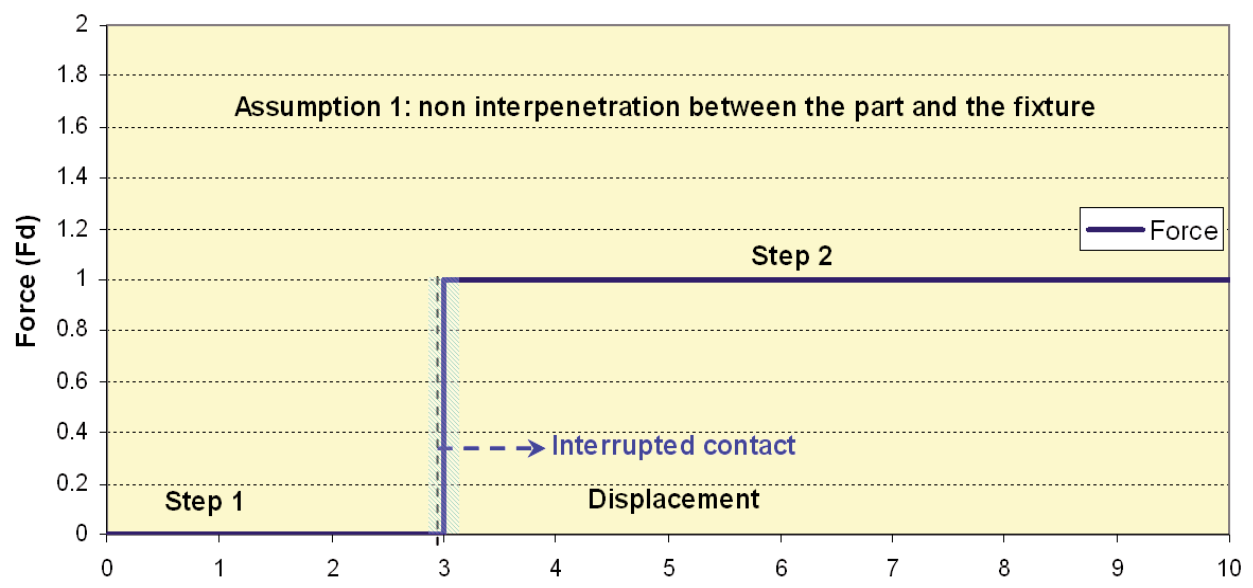

Fig. 15. The disassembly force without interpenetrations of the surfaces.

them. During the repeatability of the assembly-disassembly of the workpiece, the force $\left(\overrightarrow{F_{D}}\right)$ that needs to separate the contacts is described in 2 distinct steps:

- Step 1: Before the interruption of the contacts $\sum_{i} \overrightarrow{F_{i}}+\vec{P}=$ $\overrightarrow{0}$, does the $\overrightarrow{F_{D}}$ exist or not?

- Step 2: After the interruption of the contacts $\overrightarrow{F_{D}} \neq \overrightarrow{0}$, so $\overrightarrow{F_{D}}+\vec{P}=\overrightarrow{0}$

The plot of $\overrightarrow{F_{D}}=f(\delta) \cdot \vec{z}$ is shown as in Figure 15 .

\subsubsection{Second assumption}

The surface defects will be taken into account (Fig. 16) in this assumption.

Due to these defects, there may be interpenetrations of the surface contacts. It thus prevents the separation of the workpiece and the fixture during the disassembly, and the force that appears in this step is named binding force $\overrightarrow{F_{C}}$. Hence, there is an extra step during the disassembly, namely the transient step.

The force $\left(\overrightarrow{F_{D}}\right)$ needed to separate the contact between the workpiece and the fixture can be described in 3 distinct steps:

- Step 1: The workpiece contacts on the fixture: $\overrightarrow{F_{D}}=\overrightarrow{0}$, so $\sum_{i} \vec{F}_{i}+\vec{P}=\overrightarrow{0}$

- Step 2 (transient step): appearance of binding forces: $\overrightarrow{F_{C}} \neq$ $\overrightarrow{0}=f(\delta) \cdot \vec{z}$, so $\sum_{i} \overrightarrow{F_{C}}+\vec{P}+\overrightarrow{F_{D}}=\overrightarrow{0} ;\left(\overrightarrow{F_{D}}>\vec{P}\right)$.

- Step 3: the separation of the workpiece and the fixture: $\overrightarrow{F_{D}} \neq \overrightarrow{0}$, so $\overrightarrow{F_{D}}+\vec{P}=\overrightarrow{0}$.

In this case, the force needed to disassemble the workpiece must be greater than or equal to the binding force $\left(\overrightarrow{F_{C}} \neq \overrightarrow{0}\right)$ and the workpiece weight. Thus, the resultant force $\overrightarrow{F_{D}}$ is greater than $\vec{P}$

The plot of the disassembly force $\left(\overrightarrow{F_{D}}\right)$ is shown in Figure 17. 


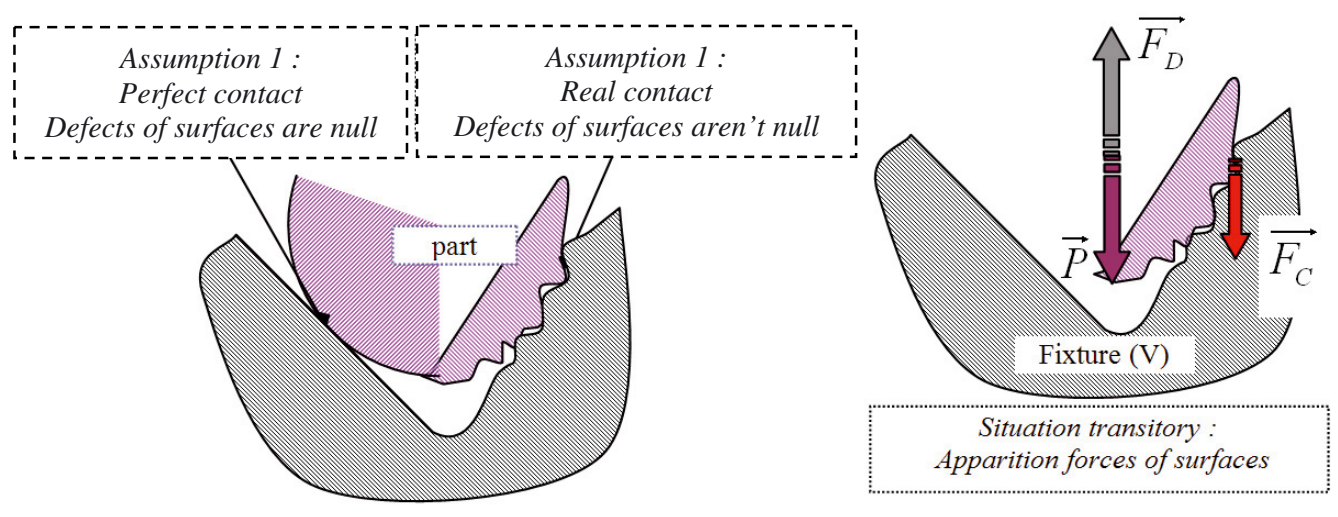

Fig. 16. The interpenetrations of the surfaces.

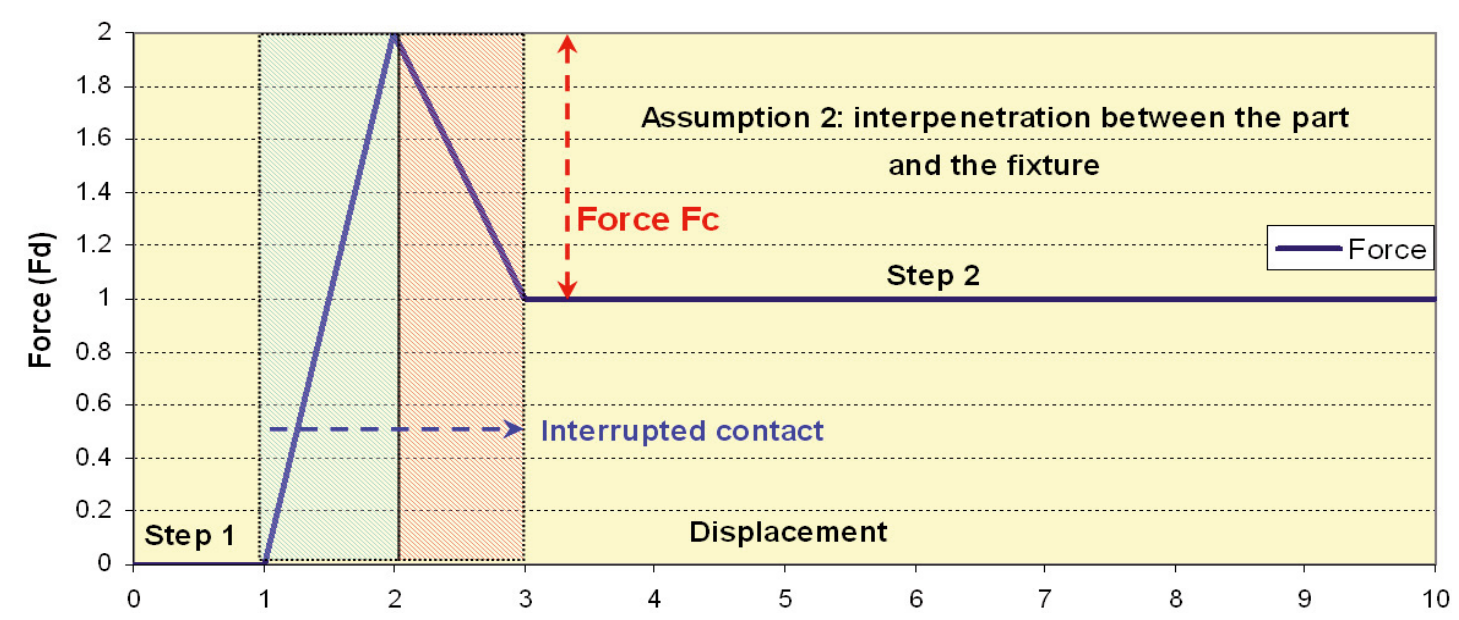

Fig. 17. The disassembly force with interpenetrations of the surfaces.

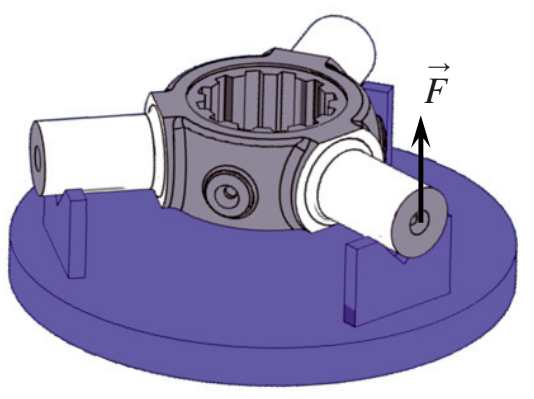

Fig. 18. Lifting force.

\subsection{Experimental validation}

To experimentally verify the presence of the binding force $\left(\overrightarrow{F_{C}} \neq \overrightarrow{0}\right)$, a series of tests was carried out on the traction machine (Instron).

\subsubsection{Description of tests}

Some of the parameters used in this test can be described as follows:
- choose a geometry of the V-Blocks $\left(90^{\circ}\right.$ and $\left.120^{\circ}\right)$,

- set the assembly process on a traction machine,

- use the vertical movement for lifting each trunnion of the workpiece,

- plot the lifting force $\vec{F}$ (Fig. 18).

\subsubsection{Results and conclusions}

The lifting force $(\vec{F}=f(\delta) \cdot \vec{z})$ was plotted after the series of measurements.

The experimental results (Fig. 19) show that:

- The presence of the binding force $\left(\overrightarrow{F_{C}}\right)$ at the contact points is right as in the second assumption.

- This force can be considered as a factor for explaining the deviation (indicators- measures) that presented in the above analyses (the first two indicators).

- There is a difference between the binding forces in the different parameters of the V-Blocks.

- $\overrightarrow{F_{C}^{V 120}}<\overrightarrow{F_{C}^{V 90}}$. It seems to respect the mechanical system analysis. Indeed, the binding force $\left(\overrightarrow{F_{C}}\right)$ depends on the angle of $\mathrm{V}$ as in Figure 20. It can be observed that $\overrightarrow{F_{C}}$ is maximum for the V0 and minimum for the V180. 


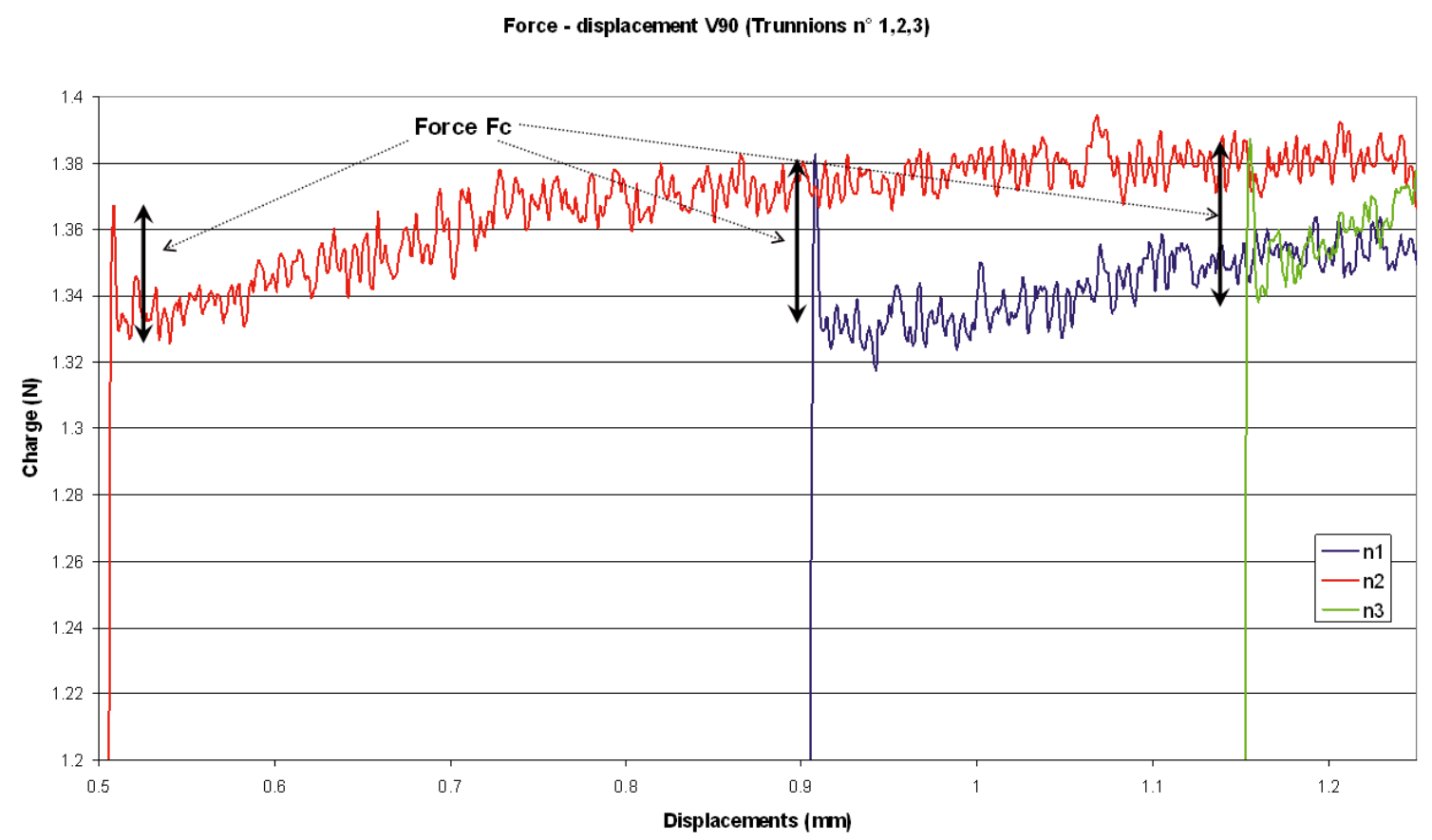

Fig. 19. Experimental plot of the lifting forces.

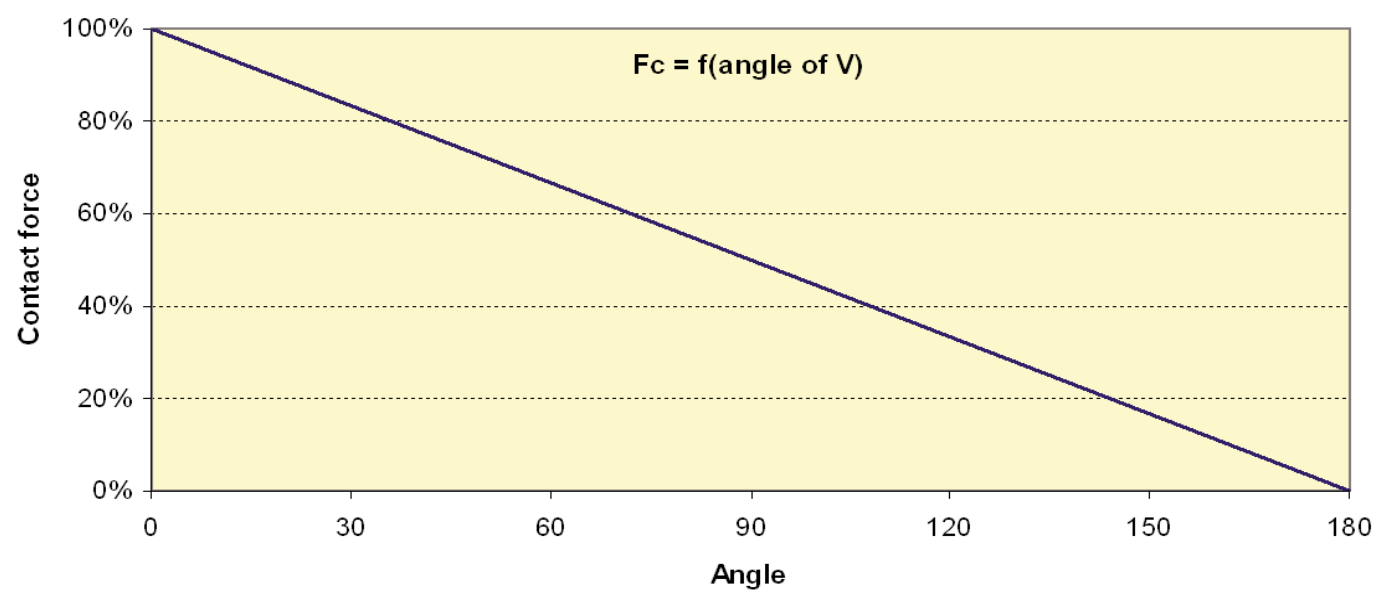

Fig. 20. Relation between the binding force and the angles of V-Block.

\section{Conclusions}

Our investigations of the indicators show that:

- The "Euclidean condition" indicator is a promising indicator that can be used to optimise the different solutions in fixture design or in the choice of fixtures in manufacturing and assembly.

- It is not enough to consider a sole geometric parameter of a fixture for evaluating the localization of a workpiece on the fixture.

- The influence of the friction force and the surface defects at the contact points is also an important factor that needs to be taken into account when controlling the quality of a fixture.
This study also allows us to confirm that the variability of isostatic localization is small.

The results show that the binding force at contacts is significant. It would be worthwhile to quantify this force more precisely. Thus, it can be better integrated into the indicator that allows estimating the quality of workpiece localizations on a fixture.

The methods that were used to analyse localization, reaction forces and frictions can be integrated into software in order to choose an optimal fixture in manufacturing, measuring or assembling.

The indicators that are proposed and validated are simple and robust and this is the principal objective of this study. Nevertheless, other indicators, more complex to implement, can be considered. 


\section{Nomenclature}

\begin{tabular}{|c|c|}
\hline - & dot product or scalar product of two vectors \\
\hline$\wedge$ & crossproduct of two vectors \\
\hline$R(O, \vec{x}, \vec{y}, \vec{z})$ & coordinate system of a fixture \\
\hline$\{\mathbf{D}\}$ & Small Displacement Torsor (SDT) \\
\hline$\left\{\begin{array}{l}\vec{D}=(u, v, w) \\
\overrightarrow{\Omega_{S / R}}=(\alpha, \beta, \gamma)\end{array}\right.$ & translations and rotations of a SDT \\
\hline$M_{i}\left(x_{i}, y_{i}, z_{i}\right)$ & contact point of workpiece-fixture pair \\
\hline $\overrightarrow{n_{i}}=\left(n_{x i}, n_{y i}, n_{z i}\right)$ & normal vector at a point contact $i$ \\
\hline$\left\{\mathbf{P}_{i}\right\}$ & torsor of the Plücker coordinates of the normal at a contact point $i$ \\
\hline $\overrightarrow{g_{O}}$ & product vector of $\overrightarrow{O M_{i}}$ and $\overrightarrow{n_{i}}$ \\
\hline$r$ & rank of a line system \\
\hline$d$ & degree of freedom \\
\hline $\mathrm{V}$ & angle of V-Block \\
\hline$\vec{P}$ & gravity of a workpiece \\
\hline $\overrightarrow{F_{i}}$ & reaction force of the workpiece on the fixture \\
\hline $\tan (\varphi)$ & friction coefficient at contact points \\
\hline $\overrightarrow{\delta_{i}}$ & displacement vector of the workpiece's contact point \\
\hline $\overrightarrow{F_{D}}$ & disassembly force \\
\hline $\overrightarrow{F_{C}}$ & binding force \\
\hline$\vec{F}$ & lifting force \\
\hline
\end{tabular}

\section{References}

1. Y. Wang, X. Chen, Q. Liu, N. Gindy, Optimization of machining fixture layout under multi-constraints, Int. J. Mach. Tools Manuf. 46, 1291 (2006)

2. Y.F. Wang, Y.S. Wong, J.Y.H. Fuh, Off-line modelling and planning of optimal clamping force for an intelligent fixturing system, Int. J. Mach. Tools Manuf. 39, 29 (1999)

3. Y. Wu, Y. Rong, W. Ma, S.R. LeClair, Automated modular fixture planning: accuracy clamping and accessibility analyses, Robot. Comput.-Integr. Manuf. 14, 17 (1998)

4. E.C. DeMeter, Restraint analysis of fixtures which rely on surface-contact, ASME J. Eng. Ind. 116, 207 (1994)

5. Z. Xiong, M.Y. Wang, Z. Li, A near-optimal probing strategy for workpiece localization, IEEE Trans. Robot. 20, 668 (2004)

6. Y.X. Chu, J.B. Gou, H. Wu, Z.X. Li, Workpiece localization algorithm: Performance evaluation and reliability analysis, J. Manuf. Syst. 18, 113 (1999)

7. B. Li, S.N. Melkote, Improved workpiece location accuracy through fixture layout optimization, Int. J. Mach. Tools Manuf. 39, 871 (1999)
8. M.Y. Wang, An optimum design for 3-D fixture synthesis in a point set domain, IEEE Trans. Robot. Autom. 16, 839 (2000)

9. A. Raghu, S.N. Melkote, Analysis of the effects of fixture clamping sequence on part location errors, Int. J. Mach. Tools Manuf. 39, 871 (1999)

10. Y.C. Chen, C.L.P. Chen, The importance of sequence in clamping prismatic workpieces in fixturing processes, in Proc. of the 1996 IEEE, Int. Conf. on Robotics and Automation ISBN, Minneapolis, MN, 1996, pp. 503-508

11. J.M. Schimmels, M.A. Peshkin, Force-Assembly with friction, IEEE Trans. Robot. Autom. 10, 465 (1994)

12. P. Bourdet, A. Clement, Controlling a complex surface with a 3 axis measuring machine, Ann. CIRP, 354 (1976)

13. P. Bourdet, Métrologie tridimensionnelle et géométrique des pièces mécaniques, Université Paris VI - ENS de Cachan, Licence de Technologie Mécanique (1998-1999)

14. P. Bourdet, A. Clement, Optimisation des montages d'usinage, Contrat de recherche DRME (1972)

15. D. Duret, Qualité de la mesure en production (Eyrolles, Paris, 2008)

16. J.D. Lee, L.S. Haynes, Finite-element analysis of flexible fixturing system, ASME J. Eng. Ind. 109, 134 (1987) 